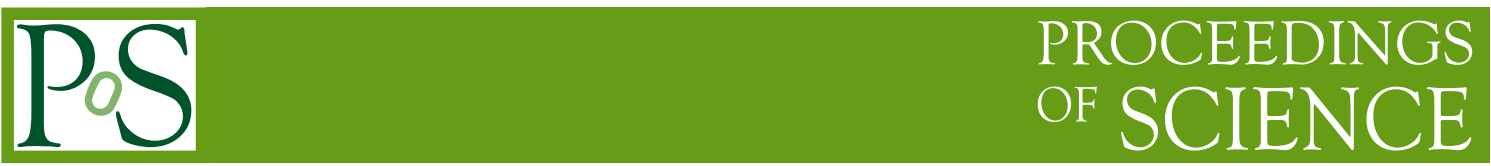

\title{
Directional dark matter search with nuclear emulsions
}

\author{
Andrey Alexandrov ${ }^{a, b, c, d, *}$ for the NEWSdm collaboration \\ ${ }^{a}$ I.N.F.N. sezione di Napoli, \\ I-80126 Napoli, Italy \\ ${ }^{b}$ Università degli Studi di Napoli Federico II, \\ I-80126 Napoli, Italy \\ ${ }^{c}$ National University of Science and Technology MISIS, \\ RUS-119049 Moscow, Russia \\ ${ }^{d}$ Lebedev Physical Institute of the Russian Academy of Sciences, \\ RUS-119991 Moscow, Russia \\ E-mail: andrey.alexandrov@na.infn.it
}

\begin{abstract}
The nature of dark matter (DM) is one of the most relevant open problems both in cosmology and particle physics. Many different experimental techniques have been developed to detect Weakly Interactive Massive Particles (WIMPs) as dark matter candidates via their scattering with detector atoms. The NEWSdm experiment, located in the Gran Sasso underground laboratory in Italy, is based on a novel nuclear emulsion technology with nanometric resolution and new emulsion scanning microscopy that can detect recoil track lengths down to one hundred nanometers. Thus, NEWSdm is able to reconstruct the direction of the nucleus recoiling, thus being capable of confirming the Galactic origin of the dark matter. We discuss the potentiality, both in terms of exclusion limits and discovery potential, of a directional experiment based on the use of a solid target made by newly developed nuclear emulsions and read-out systems reaching nanometric resolution.
\end{abstract}

40th International Conference on High Energy physics - ICHEP2020

July 28 - August 6, 2020

Prague, Czech Republic (virtual meeting)

\footnotetext{
${ }^{*}$ Speaker
} 


\section{Introduction}

Current experimental efforts for direct Dark Matter searches are devoted to the search in terrestrial underground detectors for rare interactions between galactic halo WIMPs and nuclei. Since dark matter detectors are rapidly improving their sensitivity, in the next decade they will encounter the neutrino background where Solar, atmospheric, and diffuse supernova neutrinos mimic the dark matter signal. Neutrinos are therefore the ultimate background for WIMP direct searches as they cannot be shielded and produce recoils with similar rates and energy spectra. Moreover, the controversy in the low-mass (order of $10 \mathrm{GeV} / \mathrm{c}^{2}$ ) WIMP region where some dark matter hints were shown, highlights the need for additional discrimination power between WIMP events and backgrounds to unambiguously prove WIMP signals. New generation detectors capable of measuring the direction of nuclear recoil tracks resulting from the WIMP elastic scattering off target nuclei would provide the discrimination of neutrino background and the unambiguous identification of galactic WIMPs as dark matter candidates. Several approaches have been proposed [1]. The most popular one is based on the use of low pressure gaseous Time Projection Chambers that have put the first directional limits in the Spin-Dependent case. Nonetheless, this technology is hardly scalable to very large detector masses. The use of a solid target for directional searches would instead overcome this mass limitation, thus allowing to reach a high sensitivity in the low cross-section region. Nevertheless, in a solid medium, the track length of a nuclear recoil would be of the order of a few hundred nanometers, thus requiring a detector with ultra-high tracking resolution. This is the challenge addressed by the NEWSdm proposal.

\section{The NEWSdm experiment}

The approach proposed by the NEWSdm Collaboration [2] consists of using a nuclear emulsionbased detector acting both as target and as nanometric tracking device. Nuclear emulsions are made of silver halide crystals embedded in a gelatine matrix. When ionizing particles pass through it, some of the halide crystals are modified in such a way that they are turned into grains of silver after the developing process. The three-dimensional trajectory of passing through particles can be reconstructed with an optical microscope by connecting all the silver grains. The NEWSdm project foresees the employment of a novel emulsion technology called Nano Imaging Trackers (NIT) [3, 4] with $\mathrm{AgBr}$ crystals of nanometric size, about one order of magnitude smaller than those used in the OPERA experiment. The detector is conceived as a bulk of NIT surrounded by a shield to reduce the external background. The detector is then placed on an equatorial telescope in order to absorb the Earth rotation, thus keeping fixed the detector orientation with respect to the incoming apparent WIMP flux. The angular distribution of the WIMP-scattered nuclei is therefore expected to be strongly anisotropic with a peak centered in the forward direction. NIT have a linear density of about 11 crystals $/ \mu \mathrm{m}$ [3], thus making the reconstruction of trajectories with path lengths shorter than $100 \mathrm{~nm}$ possible, if analyzed by means of microscopes with enough resolution. The presence in the emulsion gel of lighter nuclei such as carbon, oxygen and nitrogen, in addition to the heavier nuclei of silver and bromine, is a key feature of the NEWSdm project, resulting in a good sensitivity to WIMPs in the mass range between 10 to $100 \mathrm{GeV} / \mathrm{c}^{2}$. 
The NEWSdm collaboration has setup at the Gran Sasso underground laboratory a facility for the emulsion handling and film production. The experimental setup is located in the Hall B of the underground Gran Sasso Laboratory and it consists of a shield from environmental backgrounds and a cooling system to ensure the required temperature level to the NIT emulsion detector. The optimization of the shield structure, along with the definition of the material thickness, was performed with a Geant 4 simulation.

Optical microscope read-out system In the NEWS experiment a WIMP signal consists of shortpath, anisotropically distributed, nuclear recoils over an isotropically distributed background. The search for signal candidates requires the scanning of the whole emulsion volume. The read-out system has therefore to fulfill two main requirements: a fast, completely automated, scanning system is needed to analyse the target volume over a time scale comparable with the exposure; the spatial resolution has to reach the challenging value of a few tens of nanometers, well beyond the diffraction limit, in such a way to ensure high efficiency and purity in the selection of signal candidates. The analysis of NIT emulsions is performed with a two-step approach: a fast scanning with a state-ofthe-art resolution for the signal preselection followed by a pin-point check of preselected candidates with unprecedented nanometric resolution to further enhance the signal to noise ratio. In the first phase a fast scanning is performed by means of an improved version of the optical microscope used for the scanning of the OPERA films [5]. An extensive R\&D program has achieved a speed of about $200 \mathrm{~cm}^{2} / \mathrm{h}[6,7]$, which potentially can be improved by at least one order of magnitude by using the novel scanning technique developed to enable the effective use of multiple-camera setups [8].

The starting point of the emulsion scanning is the image analysis to collect clusters making up silver grains. Given the intrinsic resolution of the optical microscope $(\sim 200 \mathrm{~nm})$, the sequence of several grains making a track of a few hundred nanometers may appear as a single cluster. Nevertheless, a cluster made of several grains tends to have an elliptical shape with the major axis along the direction of the trajectory, while a cluster produced by a single grain tends to have a spherical shape. The shape analysis with an elliptical fit is indeed the first approach to select signal. In order to simulate the effect of a WIMP-induced nuclear recoil and to measure the efficiency and the resolution of the new optical prototype, a test beam with low velocity ions was performed. $\mathrm{Kr}$ ion beam with energies of 200 and $400 \mathrm{keV}$ [9] and a $\mathrm{C}$ ion beam with energies of 60, 80 and 100 $\mathrm{keV}$ were used. Silver grains belonging to the tracks appear as a single cluster. An elliptical fit of the cluster shape allows a clear separation between fog grains and signal tracks [10].

The second step of the microscope analysis makes use of the plasmon resonance effect occurring when nanometric silver grains are dispersed in a dielectric medium [11]. The polarization dependence of the resonance frequencies strongly rejects the shape anisotropy and can be used to infer the presence of non-spherical nanometric silver grains within a cluster made of several grains. NEWSdm is using this technology to retrieve track information beyond the diffraction limit. Images of the same cluster taken with different polarization angles show a displacement of the position of its barycenter. The analysis of this displacement allows to distinguish clusters made of a single grain from those made of two or more grains. To carry out such an analysis in a completely automated way, the collaboration has designed and built a dedicated super-resolution microscope [12], shown in the left panel of Fig.1, which is equipped with a polarization spectrum analyser based on liquid crystals. An unprecedented accuracy about $3 \mathrm{~nm}$ has been achieved in both coordinates with this 

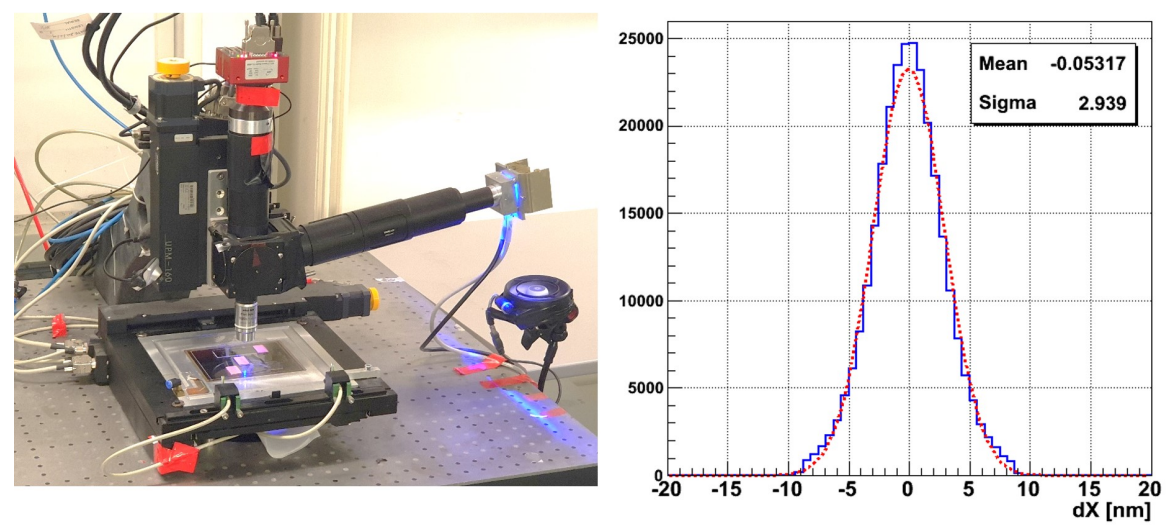

Figure 1: (left) The super-resolution microscope designed by the NEWSdm collaboration. (right) Achieved position accuracy.

microscope as shown in the right panel of Fig.1.

The peak wavelength of the scattered light depends on the size of the grains where light is scattered off. Therefore, the latest version of the optical microscope makes use of a colour camera, thus providing sensitivity to the sense of the track, since at the end of the track range grains are expected to be larger and therefore the scattered light shifts to the red colour.

Background Background sources for dark matter searches are $\alpha$ and $\beta$ particles, $\gamma$-rays and neutron induced recoils, while NIT are essentially not sensitive to minimum ionizing particles (MIP). The main sources of $\alpha$-particles are $\mathrm{U}$ and Th radioactive chains and radon. The $\alpha$-particles produced in those processes have energies of the order of $\mathrm{MeV}$ and their range in emulsion is of the order of tens of microns, by far longer than WIMP-induced nuclear recoils. $\alpha$-particles can therefore be identified and discarded in the emulsions by an upper cut on the track length. The $\beta$-rays produced in ${ }^{14} \mathrm{C}$ decay constitute a non-negligible contribution to the total background budget. This kind of background is anyway less critical for NIT emulsion with respect to other sources: electrons can be rejected by properly regulating the emulsion response, in terms of number of sensitized crystals per unit path length, through a chemical treatment of the emulsion itself. Moreover possible improvements in the rejection power can be achieved exploiting the response of $\beta$-rays to the polarized light scattering or performing a cryogenic exposure and by exploiting the phonon effect. Neutron induced recoils are the main background source because they are not distinguishable from the expected WIMP signal, except for the isotropic angular distribution and for the typical track length, largely exceeding the range expected for WIMP-induced recoils. Three types of neutron sources affect underground experiments: radiogenic neutrons in the $\mathrm{MeV}$ range produced in $(\alpha, \mathrm{n})$ and spontaneous fission reactions in the detector due to its intrinsic radioactive contaminants, cosmogenic neutrons with energy spectrum extending to $\mathrm{GeV}$ energies induced by muons penetrating underground through the rock, neutrons induced by environmental radioactivity. While the external neutron flux can be reduced to a reasonable level with an appropriate shielding, the intrinsic emulsion radioactivity would be responsible of an irreducible neutron yield through $(\alpha$, n) and ${ }^{238} U$ spontaneous fission reaction. In order to estimate this contribution, the activities of $U$ and $\mathrm{Th}$ in the emulsion components has been measured with the Inductively Coupled Plasma Mass 

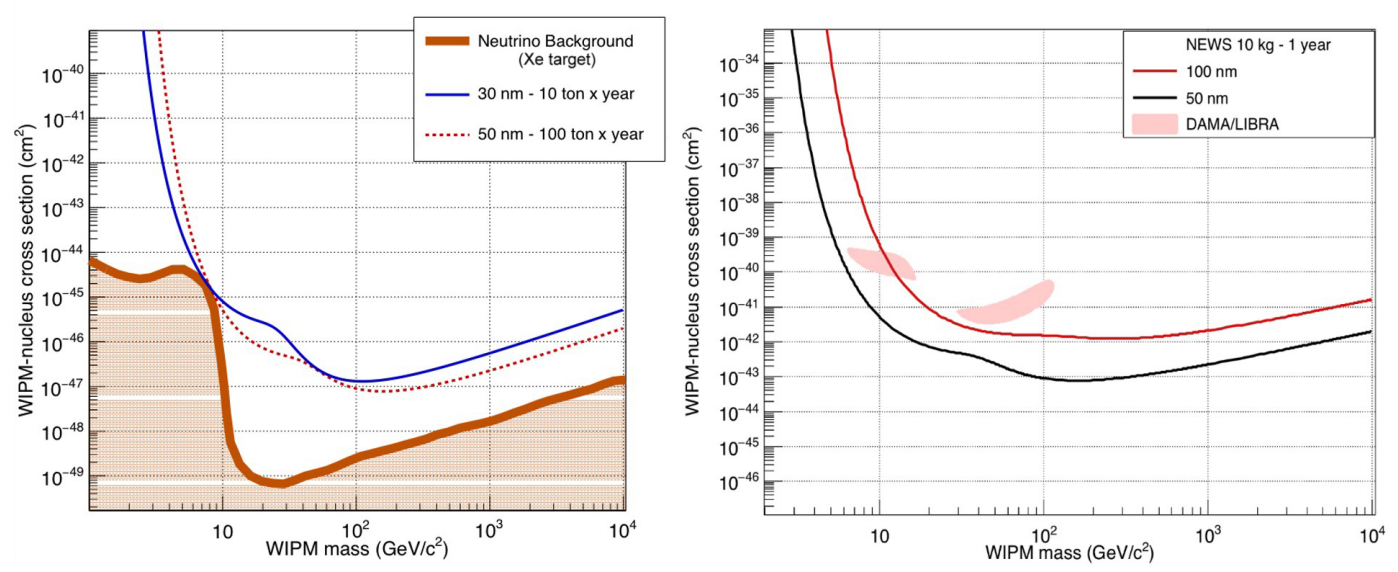

Figure 2: (left) Discovery potential of an emulsion detector with different track length thresholds and exposures. The neutrino floor, evaluated in [14] for Xe target, is also drawn for comparison. (right) Exclusion plot for the planned $10 \mathrm{~kg} \cdot y e a r$ pilot experiment.

Spectrometry (ICP-MS) and with the $\gamma$-spectrometry. The detectable neutron-induced background has been estimated to be about 0.06 per year per kilogram [13], thus allowing to design a detector for an exposure of $10 \mathrm{~kg} \cdot$ year without any further purification.

Sensitivity Left panel of Fig. 2 shows the discovery potential of an emulsion based detector. Different track length thresholds and detector exposures are assumed: the dotted red line represents a $50 \mathrm{~nm}$ threshold with 100 ton-year exposure while the solid blue line assumes a $30 \mathrm{~nm}$ threshold with an exposure of 10 ton.year. Both curves assume a negligible background. The bumps on the curves are due to the presence of light $(\mathrm{C}, \mathrm{N}$ and $\mathrm{O}$ ) and heavy ( $\mathrm{Ag}$ and $\mathrm{Br}$ ) components in the emulsion composition. The contribution from heavy nuclei dominates at high WIMP masses while light nuclei extend the sensitivity limit toward lower WIMP masses, leading to a bump in the intermediate WIMP mass range. Given the nanometric accuracy achieved and shown in Fig.1, it is realistic to assume a position resolution of several tens of nanometers provided that the grain density is sufficiently high. A mass of about 10 ton would provide sensitivity to explore the region beyond the neutrino floor shown as the thick orange curve in the same plot.

\section{Summary and outlook}

The NEWSdm experiment is meant to be the first detector with a solid target for directional dark matter searches: the use of a nuclear emulsion based detector, acting both as target and tracking device, would allow to extend dark matter searches beyond the neutrino floor and provide an unambiguous signature of the detection of Galactic dark matter. The novel emulsion technology, based on the use of nuclear emulsion films with nanometric $\mathrm{AgBr}$ crystals and of newly developed optical microscopes has achieved already an unprecedented spatial accuracy of about $3 \mathrm{~nm}$. This resolution allows to resolve grains of nanometric tracks, thus providing a very high signal to noise ratio. The current intrinsic radioactive level allows to design an experiment with a $10 \mathrm{~kg} \cdot \mathrm{year}$ exposure. A careful selection of the emulsion components and a better control of their production 
could further increase the radiopurity, thus allowing larger exposures. We plan to perform a pilot experiment with $\sim 10 \mathrm{~kg} \cdot$ year exposure with the sensitivity shown in the right panel of Fig.2, thus exploring the region indicated by the DAMA experiment with a powerful and complementary approach and demonstrating the power of this new directional technique. The subsequent step would be to further extend the detector mass and sensitivity to explore the region of the so-called neutrino floor.

\section{References}

[1] J. B. R. Battat, et al. Readout technologies for directional WIMP Dark Matter detection, Physics Reports 662 (2016) 1-46

[2] A. Aleksandrov, et al. NEWS: Nuclear Emulsions for WIMP Search, LNGS-LOI 48/15 (2016) $1-50$

[3] N. Natsume, et al. Low-velocity ion tracks in fine grain emulsion, Nucl. Instr. Meth. A $\mathbf{5 7 5}$ (2007) 439

[4] T. Naka, et al. Fine grained nuclear emulsion for higher resolution tracking detector, Nucl. Instr. Meth. A 718 (2013) 519

[5] A. Alexandrov, et al. A new fast scanning system for the measurement of large angle tracks in nuclear emulsions, JINST 10 (2015) P11006

[6] A. Alexandrov, et al. A new generation scanning system for the high-speed analysis of nuclear emulsions, JINST 11 (2016) P06002

[7] A. Alexandrov, et al. The Continuous Motion Technique for a New Generation of Scanning Systems, Scientific Reports 7 (2017) 7310

[8] A. Alexandrov, et al. A Novel Optical Scanning Technique with an Inclined Focusing Plane, Scientific Reports 9 (2019) 2870

[9] T. Naka, et al. R\&D Status of Nuclear Emulsion for Directional Dark Matter Search, EAS Publ. Ser. 53 (2012) 51

[10] N. Kimura and T. Naka, Submicron track readout in fine-grained nuclear emulsions using optical microscopy, Nucl. Instr. Meth. A 680 (2012) 12

[11] H. Tamaru, et al.. Resonant light scattering from individual Ag nanoparticles and particle pairs, Applied Phys. Lett. 80 (2002) 1826

[12] A. Alexandrov, et al. Super-resolution high-speed optical microscopy for fully automated readout of metallic nanoparticles and nanostructures, Scientific Reports 10 (2020) 18773

[13] A. Alexandrov, et al. Intrinsic neutron background of nuclear emulsions for directional Dark Matter searches, Astropart. Phys. 80 (2016) 16

[14] J. Billard, et al. Implication of neutrino backgrounds on the reach of next generation dark matter direct detection experiments, Phys. Rev. D 89 (2014) 023524 